UVX 2010 (2011) 217-220

DOI: $10.1051 /$ uvx/2011032

(C) Owned by the authors, published by EDP Sciences, 2011

\title{
Sum-frequency mixing and phase matching in a mercury ablation plume
}

\author{
L. Philippet, S. Chénais, S. Forget and M.-C. Castex* \\ Laboratoire de Physique des Lasers, C.N.R.S / Université Paris 13 Institut Galilée, \\ 99 av. Jean-Baptiste Clément 93430 Villetaneuse
}

\begin{abstract}
We report on laser-induced vaporization of mercury as a simple tool to increase the efficiency of a resonant 4-wave mixing process to produce VUV radiation near $125 \mathrm{~nm}$. The specificity of this new nonlinear medium is discussed for the best experimental conditions giving a high density of ground state $\mathrm{Hg}$ atoms in a column-shape plume. Modification of phase-matching conditions is demonstrated in agreement with VUV profile simulations.
\end{abstract}

\section{INTRODUCTION}

Metal vapors have been the first nonlinear media used for generate UV and VUV coherent light by four-wave mixing [1-3]. Among them mercury vapour is especially well suited for producing tunable radiation by a resonant sum-frequency-mixing process $\omega_{\mathrm{VUV}}=2 \omega_{\mathrm{UV}}+\omega_{\mathrm{VIS}}$. When the pump beams are used in a tightly focussed configuration, VUV generation is usually observed for wavelengths at which the nonlinear medium is negatively dispersive, i.e. for wavevector mismatch $\Delta k=k_{\mathrm{VuV}}-$ $2 k_{\mathrm{UV}}-k_{\mathrm{VIS}}<0$. In these first experiments heat pipe techniques were used to reach high $\mathrm{Hg}$ densities $\left(10^{16}-10^{17} \mathrm{at} / \mathrm{cm}^{3}\right)$ since the VUV intensity, in a phase-matched configuration and in absence of saturation effects, scales as the square of atomic density.

In the last few years [4] we have demonstrated that VUV generation around $125 \mathrm{~nm}$ enhanced both by two-photon $6 \mathrm{~s}^{1} \mathrm{~S}_{0} \rightarrow 7 \mathrm{~s}{ }^{1} \mathrm{~S}_{0}$ and three-photon $6 \mathrm{~s}^{1} \mathrm{~S}_{0} \rightarrow 9 \mathrm{p}^{61} \mathrm{P}_{1}$ resonances could be observed without heating mercury (with $\mathrm{Hg}$ vapour pressure as low as $\approx 10^{-3}$ mbar at room temperature) and with only one tunable frequency-doubled dye laser. Recently, in order to increase the VUV intensity generated with this simple experiment, we tested an original method based on laser vaporization of liquid mercury [5], which enabled the VUV intensity to be enhanced by up to two orders of magnitude. In this paper we report on the experimental characterization of the nonlinear medium, and provide evidence of phase-matching modification in a laser ablation plume, probed by the VUV generation on both sides of the $9 \mathrm{p}{ }^{1} \mathrm{P}_{1}$ atomic line in positive and negative dispersion regions.

\section{EXPERIMENTAL SETUP AND ABLATION PLUME CHARACTERISTICS}

The non-amplified VUV source at $125 \mathrm{~nm}$ has been described in detail in previous papers [4,5]. The amplified source is represented in fig. 1. The two collinear laser beams at $626 \mathrm{~nm}\left(\omega_{\text {VIS }}\right)$ and $313 \mathrm{~nm}$ $\left(\omega_{\mathrm{UV}}\right)$ are focussed at a distance $h$ above a liquid mercury pool confined at room temperature (n $\left.\approx 4.510^{13} \mathrm{at} / \mathrm{cm}^{3}\right)$ in a cell $(\mathrm{L}=30 \mathrm{~cm}$; either vacuum-pumped or filled with a neutral gas). An ArF excimer laser $(3 \mathrm{~mJ}, 4 \mathrm{~ns} / \mathrm{pulse}, 10 \mathrm{~Hz})$ is more or less focused onto the liquid mercury (different $\Delta \mathrm{f}$ values, $\Delta \mathrm{f}=0$ corresponds to the focal point onto the pool surface and $\Delta \mathrm{f}=15$ is a focalisation $15 \mathrm{~mm}$ above the surface). The plume expansion (in vacuum or in buffer gas) is highly dependent on the ArF

*e-mail: marie-claude.castex@univ-paris13.fr

This is an Open Access article distributed under the terms of the Creative Commons Attribution-Noncommercial License 3.0, which permits unrestricted use, distribution, and reproduction in any noncommercial medium, provided the original work is properly cited. 


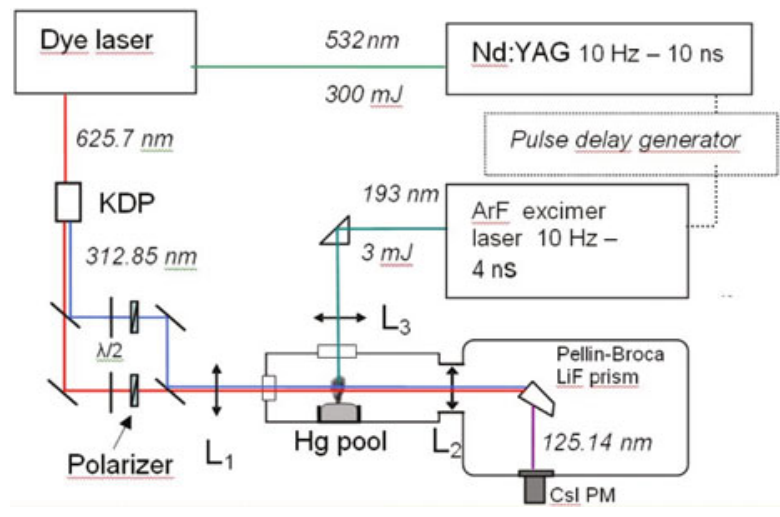

Figure 1. Experimental setup.

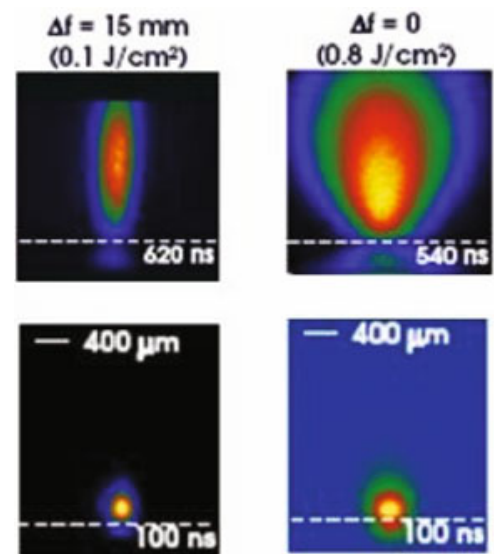

Figure 2. $\mathrm{Hg}$ plume expansion in vacuum as a function of ArF focusing and time.

laser focusing conditions. The plume obtained for tight focusing $(\Delta \mathrm{f}=0)$ was found to have a wider angular spreading than the "column-shaped" plume obtained for $\Delta \mathrm{f}=15 \mathrm{~mm}$ (Fig. 2). The time delay between the ArF excimer pulse and the two UV and VIS pulses producing 4-wave mixing constitute also an important parameter linked to the density and the nature of species ejected in the plume. The dense vapor plume has been investigated through time-resolved imaging (with an Andor iStar DH734 camera) as well as by spectroscopy (with use of an Andor iStar DH720 camera coupled with a Jobin Yvon SPEX 270 M monochromator). Spacially-resolved imaging and time-resolved spectral analysis of the plume emission is necessary to understand dynamic expansion of the different $\mathrm{Hg}$ species ejected.

Compared to VUV generation in a room-temperature $\mathrm{Hg}$ cell, a significant enhancement of the VUV intensity was observed thanks to vaporisation. It is found that VUV intensity is maximized for a $700 \mathrm{~ns}$ time delay between the ArF excimer pulse and the two UV and VIS pulses producing 4-wave mixing. Once the best experimental conditions for $\mathrm{Hg}$ plume expansion were selected, an important parameter to be determined is the $\mathrm{Hg}$ atomic density $\mathrm{N}(\mathrm{z})$ inside the column-shape plume. To realize the estimation of absolute $\mathrm{N}(\mathrm{z})$ density in a $1 \mathrm{~mm}$ width $\mathrm{Hg}$ plume, we have modified our experiment to generate VUV with collimated UV and VIS beams of $1 \mathrm{~mm}$ diameter size.

\section{PLANE WAVE 4-WAVE MIXING IN MERCURY PLUME}

In the plane-wave limit VUV intensity is dependent on a phase matching sinc function

$$
\mathrm{F}\left(\mathrm{S}(\mathrm{z}), \Delta \mathrm{k}_{0}\right) \sim\left[\sin \left(\Delta \mathrm{k}_{0} / 2 \int \mathrm{S}(\mathrm{z}) \mathrm{dz}\right) / \Delta \mathrm{k}_{0} / 2 \int \mathrm{S}(\mathrm{z}) \mathrm{dz}\right]^{2}
$$

where $\mathrm{S}(\mathrm{z})$ is the density profile inside the plume and $\Delta \mathrm{k}_{0}$ is the wave vector mismatch related to the propagation of beams in the room temperature cell $\left(\mathrm{N}_{\mathrm{Hg}}=4.5210^{13} \mathrm{at} / \mathrm{cm}^{3}\right)$. The profile $\mathrm{S}(\mathrm{z})$ was measured independently by an imaging technique and is close to a "top-hat" profile of $1 \mathrm{~mm}$ width. The collimated beam arrangement allows a direct comparison between VUV intensity created either in the cell or in the plume. Moreover because of the low intensity of pump beams this configuration avoids any saturation effect as shown in fig. 3 by the fact that $I_{\mathrm{VUV}}$ scales as $I_{\mathrm{UV}}^{2}$ as expected (Fig. 3).

For an ArF vaporizing laser fluence of $1 \mathrm{~mJ}$ incident onto the pool (spot area $1 \mathrm{~mm}^{2}$ ), a ratio of 5 is deduced from the slopes of the experimental results. From the comparison with the sinusoidal gain calculated as a function of $S_{p}$ (the atomic density at the plume center relative to the background value), a 


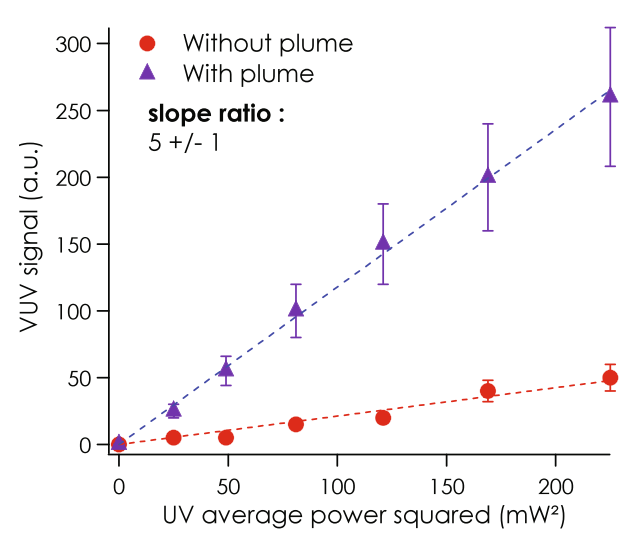

Figure 3. Comparison between $I_{\mathrm{VuV}}$ created with and without vaporisation plume, in a plane-wave configuration.

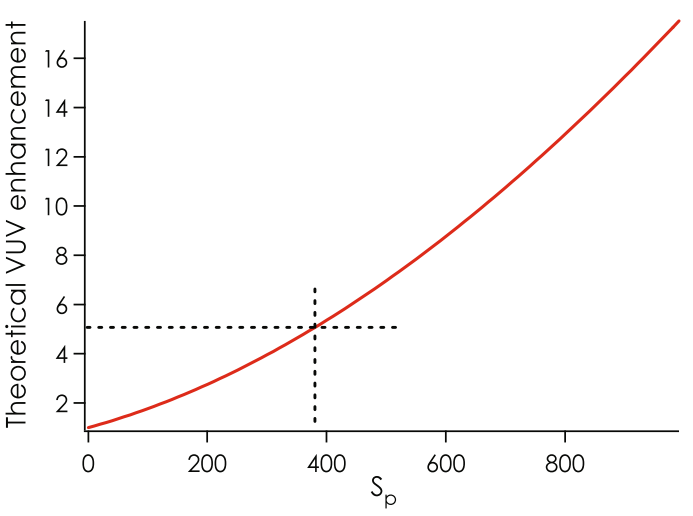

Figure 4. Theoretical gain as a function of $\mathrm{Sp}$ (relative plume density at the center of the plume with respect to the background density at room temperature). Graphical resolution gives $\mathrm{Sp} \approx 400$. The theoretical behaviour is a sinusoidal curve but any higher value of $\mathrm{Sp}$ can be ruled out as being unrealistically high in our system.

value $\mathrm{S}_{\mathrm{p}} \approx 400$ has been found (Fig. 4) which corresponds to $\mathrm{N}=\mathrm{S}_{\mathrm{p}} \mathrm{N}_{0}=1.810^{16} \mathrm{at} / \mathrm{cm}^{3}$. This density would correspond to a local heating of the interaction zone of $110^{\circ} \mathrm{C}$.

The plane-wave approximation can then be used to account for the specific behaviour of VUV generated in negative as well as positive dispersion region. An example is given by the VUV created in the vicinity of the $9 \mathrm{p}^{1} \mathrm{P}_{1} \rightarrow 6 \mathrm{~s}^{1} \mathrm{~S}_{0}$ resonance. By scanning the dye laser and thus detuning the UV beam from the 2 photon $6 \mathrm{~s}^{1} \mathrm{~S}_{0} \rightarrow 7 \mathrm{~s}{ }^{1} \mathrm{~S}_{0}$ resonance, in the homogeneous vapour cell experiment VUV was only observed on the high energy side of the $9 \mathrm{p}{ }^{1} \mathrm{P}_{1}$ resonance (Fig. 5). The VUV emission profile can be fitted assuming an oscillator strength $\mathrm{f}=0.04[6]$ for the $9 \mathrm{p}^{1} \mathrm{P}_{1} \rightarrow 6 \mathrm{~s}^{1} \mathrm{~S}_{0}$ transition and a $2 \mathrm{~cm}^{-1}$ linewidth.

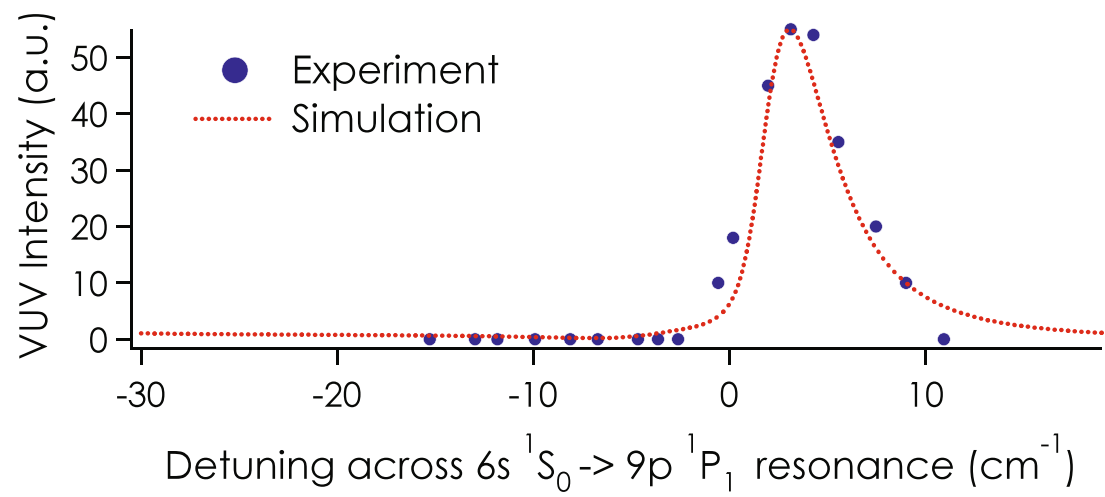

Figure 5. VUV intensity generated at the vicinity of the three-photon resonance, without ablation plume. The experimental profile was fitted with oscillator strengths taken from [6] and with the linewidth of the 9p line taken to be $2 \mathrm{~cm}^{-1}$ (fitting parameter).

In the case of Hg vaporization, VUV radiation is created on both side of resonance (Fig. 6). On the high energy side a VUV intensity enhancement of $\approx 4$ is estimated. To be able to perform a simulation of the VUV emission we have used the estimated value $\mathrm{N}=\mathrm{S}_{\mathrm{p}} \mathrm{N}_{0}=1.810^{16} \mathrm{at} / \mathrm{cm}^{3}$ for the $\mathrm{Hg}$ density inside the vaporisation plume. 


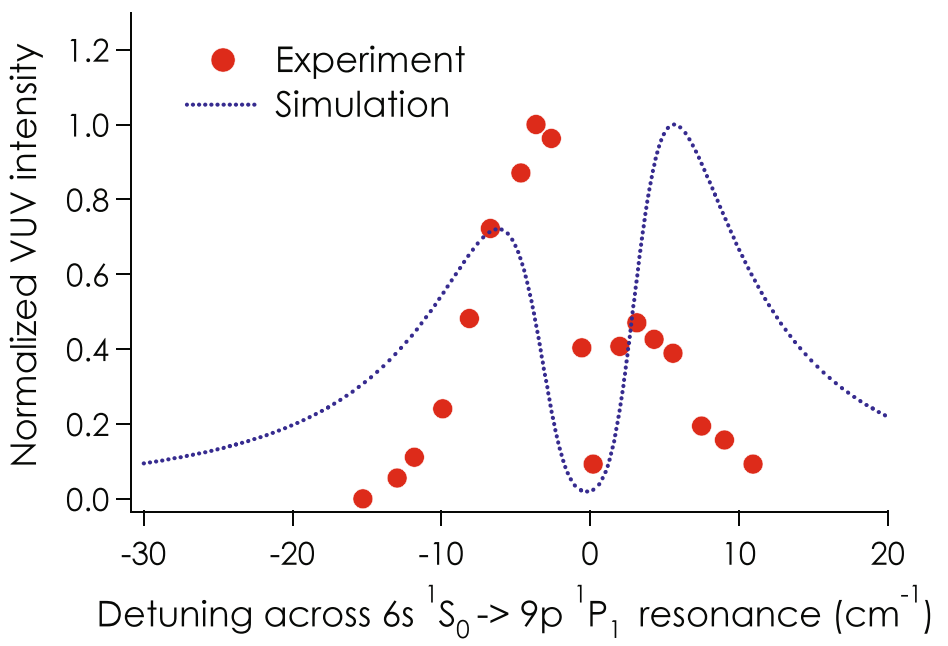

Figure 6. VUV intensity generated at the vicinity of the three-photon resonance, with ablation plume. Dotted line: theoretical simulation (without further fitting parameter than those taken in fig. 5).

Our simulation is in coarse agreement with the experimental profile and shows the modification of phase matching introduced by $\mathrm{Hg}$ vaporization. This "nonlinear medium", whose properties are largely user-controllable, can be helpful to enlarge a VUV bandwidth or when a specific nonlinear medium with a low vapour pressure has to be used. Furthermore, laser vaporisation is an easier technique than beam experiment [7].

\section{CONCLUSION}

The feasibility to use a laser-induced ablation plume of mercury as a convenient nonlinear medium is demonstrated. We have studied the intensity increase at $125 \mathrm{~nm}$ as a function of various experimental parameters. For mercury vaporization in a column-shape plume we observe VUV radiation on both sides of the atomic $6 \mathrm{~s}^{1} \mathrm{~S}_{0} \rightarrow 9 \mathrm{p}^{1} \mathrm{P}_{1}$ resonance. Modifications of phase-matching conditions agree with the profile simulations performed in positive and negative dispersion regions in the plane wave configuration.

\section{Acknowledgment}

We gratefully acknowledge Andor Technology Inc. for loaning us the camera used for imaging experiments.

\section{References}

[1] W. Jamroz, B.P. Stoicheff, Progress in Optics, 20, 326-380 (1983)

[2] R. Hilbig, R. Wallenstein, IEEE J. QE-19 1759-1770 (1983)

[3] C.H. Muller, D.D. Lowenthal, M.A. Defaccio and A.V. Smith, Opt Lett, 13, 651 (1988)

[4] L. Museur, W.Q. Zheng, A.V. Kanaev, M.C. Castex, IEEE J. Sel. Top. Quantum Electron. 1, 900 (1995)

[5] S. Chenais, S. Forget, L. Philippet, M.C Castex, Appl. Phys. B, 89, 223-229 (2007)

[6] R. Mahon and F. Tomkins, IEEE Journ. Quant. Elec. 19 (12), 1759-1770 (1983)

[7] R. Hector, K.H. Meiwes-Broer, Opt. Com. 123, 155-160 (1996) 